\title{
High-Frequency Modulated Photoluminescence: a simulation study of cases describing the signature of carrier recombination and trap centers
}

\author{
Baptiste Bérenguier ${ }^{\mathrm{a}, \mathrm{b}}$, Nicolas Moron ${ }^{\mathrm{a}, \mathrm{c}}$,Wei Zhao ${ }^{\mathrm{a}}$, Jean François Guillemoles ${ }^{\mathrm{a}, \mathrm{b}}$, Jean-Paul Kleider ${ }^{\mathrm{a}, \mathrm{c}}$, \\ Laurent Lombez ${ }^{\mathrm{a}, \mathrm{b}}$
}

\begin{abstract}
a) Institut Photovoltaïque d'Ile de France, 30 Route Départementale 12891120 Palaiseau, France
b) CNRS, UMR IPVF 9006, 30 Route Départementale 12891120 Palaiseau, France

c) GeePs, UMR 8507-CNRS, CentraleSupélec, Univ. Paris-Sud, Université Paris-Saclay, Sorbonne

Université, 11 rue Joliot-Curie, F-91192 Gif-surYvette Cedex, FRANCE
\end{abstract}

\begin{abstract}
A new setup for recording time dependent photoluminescence signal in both transient and modulated regime was previously assembled. The modulated photoluminescence experiment was achieved in a high frequency domain (up to 10 MHz) while keeping high sensitivity. The methods can thus be applied both to silicon and thin film absorbers. A simulation program was developed for the reconstruction of the experimental data in both time resolved and frequency resolved regimes. In this work we focus on the simulation part and discuss the ability of our setup to identify the carrier recombination paths in thin film solar cells. First results confirm that it should be possible to discriminate between different mechanisms such as radiative, non-radiative recombination and trapping.

Index Terms - charge carrier lifetime, photoluminescence, photovoltaic cells, semiconductor recombination, thin films absorbers.
\end{abstract}

\section{INTRODUCTION}

Thin film solar absorbers are promising candidates for the fabrication of low cost solar panels. However, the physics of some materials like CIGS or Perovskites is not completely understood. Even III-V materials can present defects having negative impacts on the efficiency by modifying the recombination paths of photo-generated carriers in the absorber volume or boundaries. Contactless methods for the characterization of these recombination paths are needed and have to be suitable to test samples at each step of the fabrication process. Photoluminescence (PL) is a powerful tool and Time Resolved Photoluminescence (TRPL) is commonly used to study recombination dynamics. However, results are often not sufficient to discriminate all the recombination mechanisms. Here, we present a complementary approach: High-Frequency Modulated Photoluminescence (HF-MPL). The experimental results are compared to the ones observed with TRPL.

First results were recently demonstrated on CIGS absorbers without and with $\mathrm{CdS}$ overlayer up to $10 \mathrm{MHz}$ modulation frequency [1]. On raw absorbers, the method gave meaningful information whereas the TRPL decay rates were too fast for suitable interpretation. However, samples with CdS covering provided complementary information to TRPL decays. It should be noted that, with TRPL, the information on time scale and injection levels are correlated, i.e. shortest times are always seen at the highest injection levels.
Although TRPL and HF-MPL data contain information on the recombination paths, the interpretation of these data is not straightforward. One needs to build a mathematical model of the sample and the usual approach is to simulate the experiment and to fit the experimental data by varying the model parameters. Simple analytic models are quite often not available whereas heavy multidimensional $(2 \mathrm{D} / 3 \mathrm{D})$ numerical simulations are time consuming and involve many parameters. Therefore, we developed a one dimensional simulation code based on drift diffusion model and including carrier trapping. The program can simulate the carriers' behavior in a multilayer stack. The specificity of this code is that the generation rate can follow any arbitrary time-dependent function. A large signal sinusoidal illumination as in HF-MPL experiments or a pulsed illumination as in TRPL can be modelled.

In this work, we present simulation results based on this code to highlight the capability of HF-MPL. By studying different cases corresponding to several recombination mechanisms, we will discuss the ability of HF-MPL to distinguish between them. In each case, a comparison with TRPL is done.

\section{METHODOLOGY}

Our drift-diffusion code was developed following a classical Gummel-Scharfetter discretization scheme [2] [3].Then, carrier capture and emission from defects were added in a similar way as in SCAPS [4] or AFORS-HET [5] following rate equations [6]. The main difference with those two wellknown programs is that we can chose an arbitrary time and depth dependent generation profile.

Fig. 1 presents our simulation methodology. The simulation starts with the calculation of the structure band diagram and the equilibrium concentration (Fig. 1.A). Then the temporal problem is solved at one frequency, waiting for the end of the transient part up to a quasi steady state and the photoluminescence is calculated (Fig. 1.B). Finally, this procedure is repeated at several frequency points. At each point, the continuous part, the phase and amplitude of the first harmonic (and also the second and third ones deduce by Fourier Transform) are extracted (Fig. 1.C). Phase and amplitude may 
provide complementary information to discriminate between recombination paths.

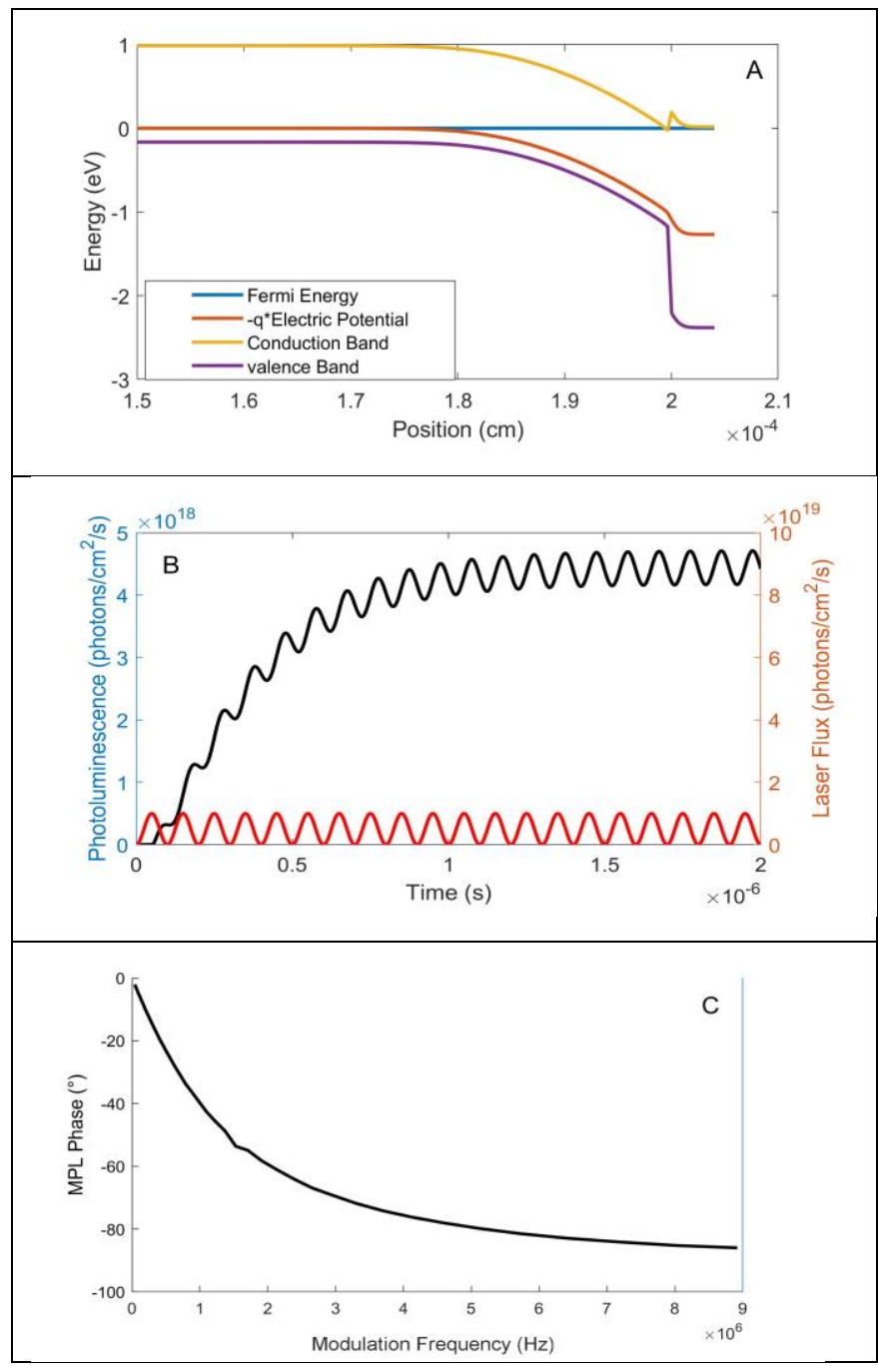

Fig. 1. An example of our simulation methodology: Equilibrium band diagram (A) of a CIGS/CdS stack, Photoluminescence response to a sinusoidal illumination (B), Extracted phase of the resulting sinusoidal signal at several frequencies $(\mathrm{C})$.

\section{SIMULATIONS}

We will consider now the simulation results obtained based on an absorber with a bandgap value of $1.2 \mathrm{eV}$. The parameters summarized in table I could correspond to CIGS, but the same methodology holds for different PV absorbers. Simulated data for four recombination mechanisms are shown on Fig. 2 to 5 . The modulation depth in these simulation is constantly $100 \%$, meaning that the continuous part of the light flux is equal to the amplitude of the modulation. Fig. 2 accounts for a single layer where only radiative recombination occurs. Fig. 3 and 4 correspond to the presence of a shallow trap close to conduction band, and to a deep mid-gap recombination center respectively. In the case of Fig. 5, all these phenomena are taken into account simultaneously.

TABLE I

SUMMARY OF MATERIAL PARAMETERS USED FOR FIG. 2 TO 5

\begin{tabular}{|l|l|}
\hline Layer thickness & $2.3 \mu \mathrm{m}$ \\
\hline Bandgap & $1.2 \mathrm{eV}$ \\
\hline P-type doping $\mathbf{N}_{\mathbf{a}}$ & $3 \mathrm{x} 10^{16} \mathrm{~cm}^{-3}$ \\
\hline Relative permittivity $\mathbf{\varepsilon}_{\mathbf{r}}$ & 13.6 \\
\hline Electron affinity & $4.1 \mathrm{eV}$ \\
\hline Electron mobility $\boldsymbol{\mu}_{\mathbf{n}}$ & $50 \mathrm{~cm}^{2} \cdot \mathrm{V}^{-1} \cdot \mathrm{s}^{-1}$ \\
\hline Hole mobility $\boldsymbol{\mu}_{\mathbf{p}}$ & $20 \mathrm{~cm}^{2} \mathrm{~V}^{-1} \mathrm{~s}^{-1}$ \\
\hline $\begin{array}{l}\text { Conduction and Valence } \\
\text { band density } \\
\text { of state } \mathbf{N}_{\mathbf{c}} / \mathbf{N}_{\mathbf{v}}\end{array}$ & $20^{18} \mathrm{~cm}^{-3}$ \\
\hline $\begin{array}{l}\text { Radiative recombination } \\
\text { coefficient }\end{array}$ & $\mathrm{K}=8 \mathrm{x} 10^{-11} \mathrm{~cm}^{3} / \mathrm{s}$ \\
\hline $\begin{array}{l}\text { Deep trap parameters } \\
\text { Shallow electron trap } \\
\text { parameters }\end{array}$ & $\begin{array}{l}\mathrm{Ec}-\mathrm{Et}=0,6 \mathrm{eV} \\
\sigma \mathrm{n}=\sigma \mathrm{p}=10^{-15} \mathrm{~cm}^{2} \\
\mathrm{Nt}=10^{17} \mathrm{~cm}^{-3}\end{array}$ \\
\hline $\begin{array}{l}\mathrm{Ec}-\mathrm{Et}=0,18 \mathrm{eV} \\
\sigma \mathrm{n}=10^{-15} \mathrm{~cm}^{2} \\
\sigma \mathrm{p}=10^{-18} \mathrm{~cm}^{2} \\
\mathrm{Nt}=10^{17} \mathrm{~cm}^{-3}\end{array}$ \\
\hline
\end{tabular}

\section{A. Radiative recombination}

Fig. 2 represents the MPL and TRPL simulations for the case of a single layer where only transport and radiative recombination occur. In this case, the material re-emits the same amount of photons that it received from the excitation, which appears quite high photoluminescence. The graph A shows the continuous part of the signal where $\mathrm{C}$ and $\mathrm{D}$ show the amplitude and phase of the first harmonic, respectively.

On Fig. 2.D, we can notice that the phase shifts towards high frequencies when increasing the illumination power. Fig. 2.E and 2.F represent the TRPL experiment for the same simulation parameters. At the two highest injection level one can see the bimolecular recombination carving the decay whereas the lower part of these decays is logarithmic, corresponding to the monomolecular regime. In the case of the HF-MPL, the bimolecular regime leads to a shift of the phase curve toward high frequencies. Fig. 2.D and 2B clearly depict the shift of the phase occurs when the injection level overcomes the doping.

\section{B. Radiative recombination + trapping.}

In Fig. 3. compared to the previous case, the main difference is the appearance for the phase curves of $\mathrm{V}$-shaped patterns (Fig. 3D). The existence of such patterns has been shown experimentally by the authors in [1]. This kind of shape would be equivalent to the bi-exponential decays in TRPL. However, the link between the trap parameters and the appearance of the 
$\mathrm{V}$-shape remains unclear. We are currently investigating this by studying the exchange rates from conduction band to the defect and from valence band to the defect. When the injection rates become high enough, all the defects are occupied, where the mentioned effect disappears. The TRPL decays are composed of a fast and a really slow part indicating the presence of two mechanisms.

\section{Radiative recombination + deep recombination center}

As observed in Fig. 4., the defect this time is no longer a shallow trap but a deep recombination center, located at midgap with important capture cross sections for both type of carriers. The corresponding electron and hole Shockley Read Hall (SRH) lifetimes would be equal to one nanosecond. This reduces drastically the carrier density and leads to a signal drop of approximatively two orders of magnitude compared to the radiative case. The phase has also been strongly reduced but remain a relevant indication of the lifetime in the material. The phase absolute value is increasing monotonically with illumination. The TRPL decays look mono-exponential like whereas the HF-MPL curves allow to distinguish the low and high injection regimes.

D. Radiative recombination + trapping + deep recombination center

In Fig. 5, It is remarkable that despite the strong recombination center, the V-shape is still present on the phase curves. The TRPL decays become bi-exponential. Both signals furnish the evidence of the presence of two mechanisms. However, it will be of great interest to perform crossing MPL and TRPL experiments and to verify the set of parameters by fitting both methods with the same set.

\section{CONCLUSION}

We have previously presented a new temporal photoluminescence method based on sinusoidal illumination: the HF-MPL. We now focus on the modelling and simulation of the experiment data, in order to estimate the ability of the method for detecting and characterizing recombination and trapping mechanisms in probed materials. We proved that the radiative recombination, shallow traps, and deep recombination centers have different signatures, both in HFMPL and TRPL which can be a useful way for material characterization. Our experimental setup is currently being upgraded to reach $100 \mathrm{MHz}$ of modulation.

\section{AKNOWLEDGMENT}

This work was supported by the French government in the frame or the program of investments for the future (Programme d'Investissement d'Avenir ANR-IEED-002-01).

\section{REFERENCES}

[1] B. Bérenguier et al., «Defects characterization in thin films photovoltaics materials by correlated high-frequency modulated and time resolved photoluminescence: An application to $\mathrm{Cu}(\mathrm{In}, \mathrm{Ga}) \mathrm{Se} 2$ », Thin Solid Films, nov. 2018.

[2] S. Ibrahim, «Modélisation et caractérisation des cellules photovoltaïques à haut rendement à base d'hétérojonctions combinant silicium cristallin et couches minces polymorphes», Thesis, Paris 11, 2010.

[3] P. Farrell, N. Rotundo, D. Hai Doan, M. Kantner, J. Fuhrmann, et T. Koprucki, « Numerical methods for drift-diffusion models », Weierstraß-Institut für Angewandte Analysis und Stochastik, 2016. http://www.wias-erlin.de/preprint/2263/wias_ preprints_2263.pdf.

[4] M. Burgelman, P. Nollet, et S. Degrave, « Modelling polycrystalline semiconductor solar cells », Thin Solid Films, vol. 361-362, p. 527-532, févr. 2000.

[5] R. Varache, C. Leendertz, M. E. Gueunier-Farret, J. Haschke, D. Muñoz, et L. Korte, «Investigation of selective junctions using a newly developed tunnel current model for solar cell applications », Sol. Energy Mater. Sol. Cells, vol. C, n 141, p. 14-23, 2015.

[6] M. Maiberg, T. Hölscher, S. Zahedi-Azad, et R. Scheer, « Theoretical study of time-resolved luminescence in semiconductors. III. Trap states in the band gap », J. Appl. Phys., vol. 118, $\mathrm{n}^{\circ} 10$, p. 105701, sept. 2015. 

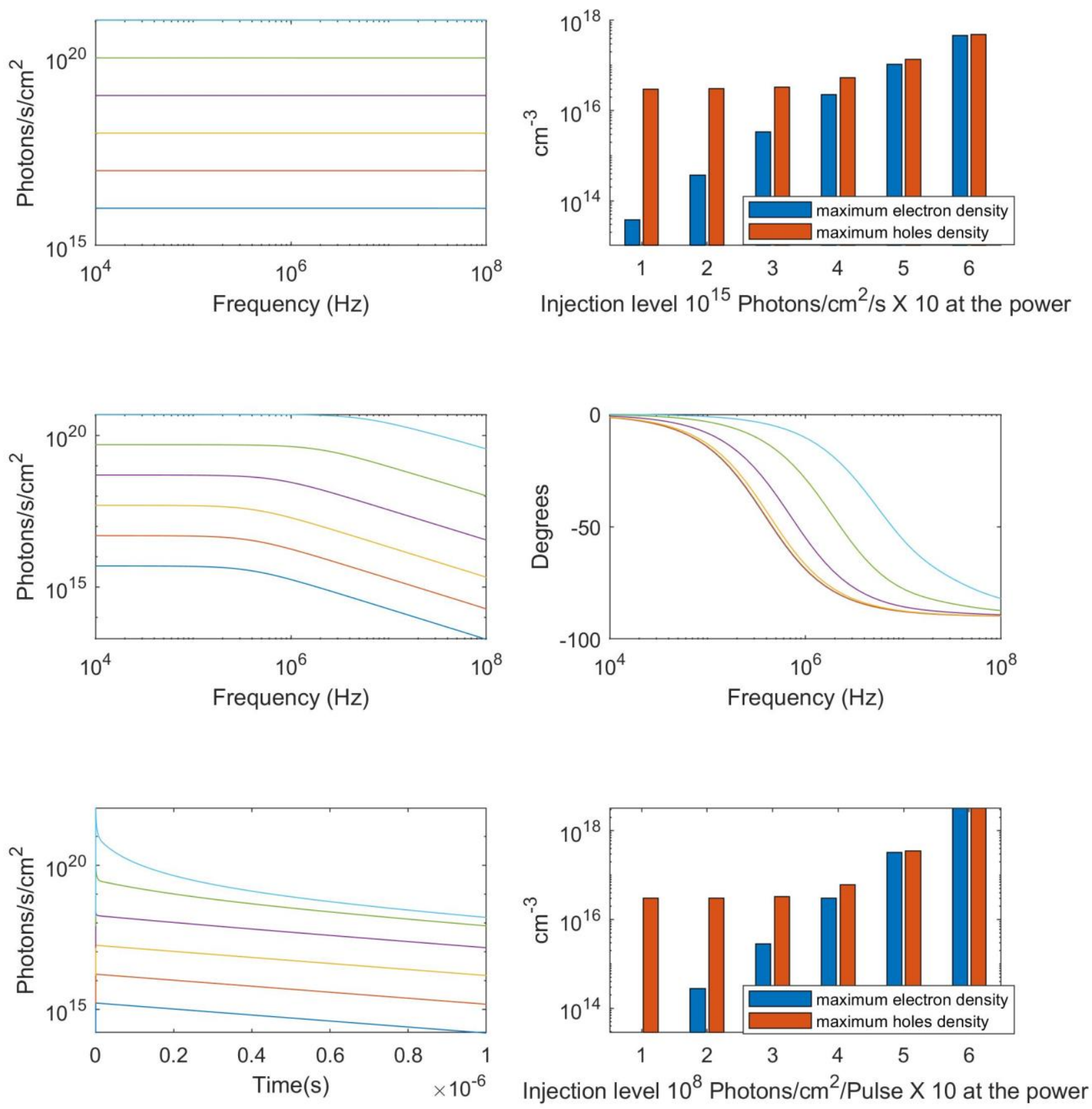

Fig. 2. Radiative recombination. From A to D: simulated HF-MPL: A continuous part, B Injection level for electrons (blue) and holes (red), C first harmonic amplitude; D first harmonic phase at several values of the average excitation flux: $10^{15}$ photons $/ \mathrm{cm}^{2} / \mathrm{s}$ multiplied by ten at the power 1 (dark blue curve), 2 (red curve), 3 (yellow curve), 4 (purple curve), 5 (green curve), 6 (light blue curve). E and F: E simulated TRPL and F corresponding injection level corresponding to the pulsed excitation flux $10^{8}$ photons $/ \mathrm{cm}^{2} /$ pulse multiplied by ten at the power 1 (dark blue curve), 2 (red curve), 3 (yellow curve), 4 (purple curve), 5 (green curve), 6 (light blue curve). 

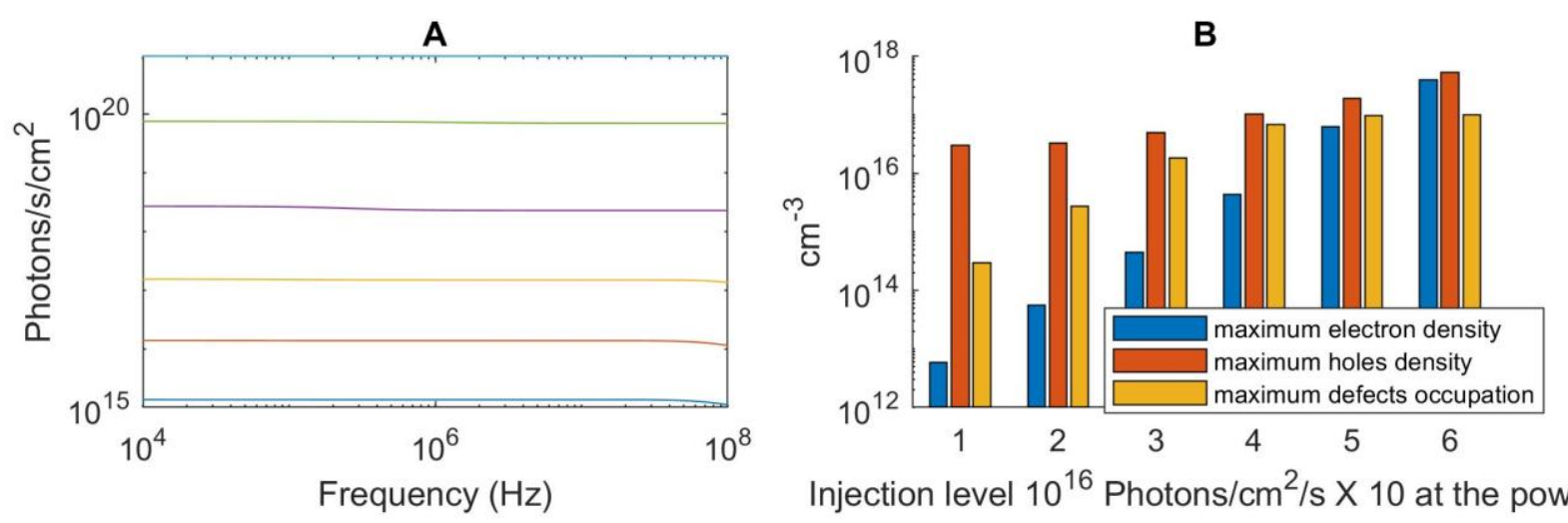

\section{Injection level $10^{16}$ Photons $/ \mathrm{cm}^{2} / \mathrm{s} \times 10$ at the power}
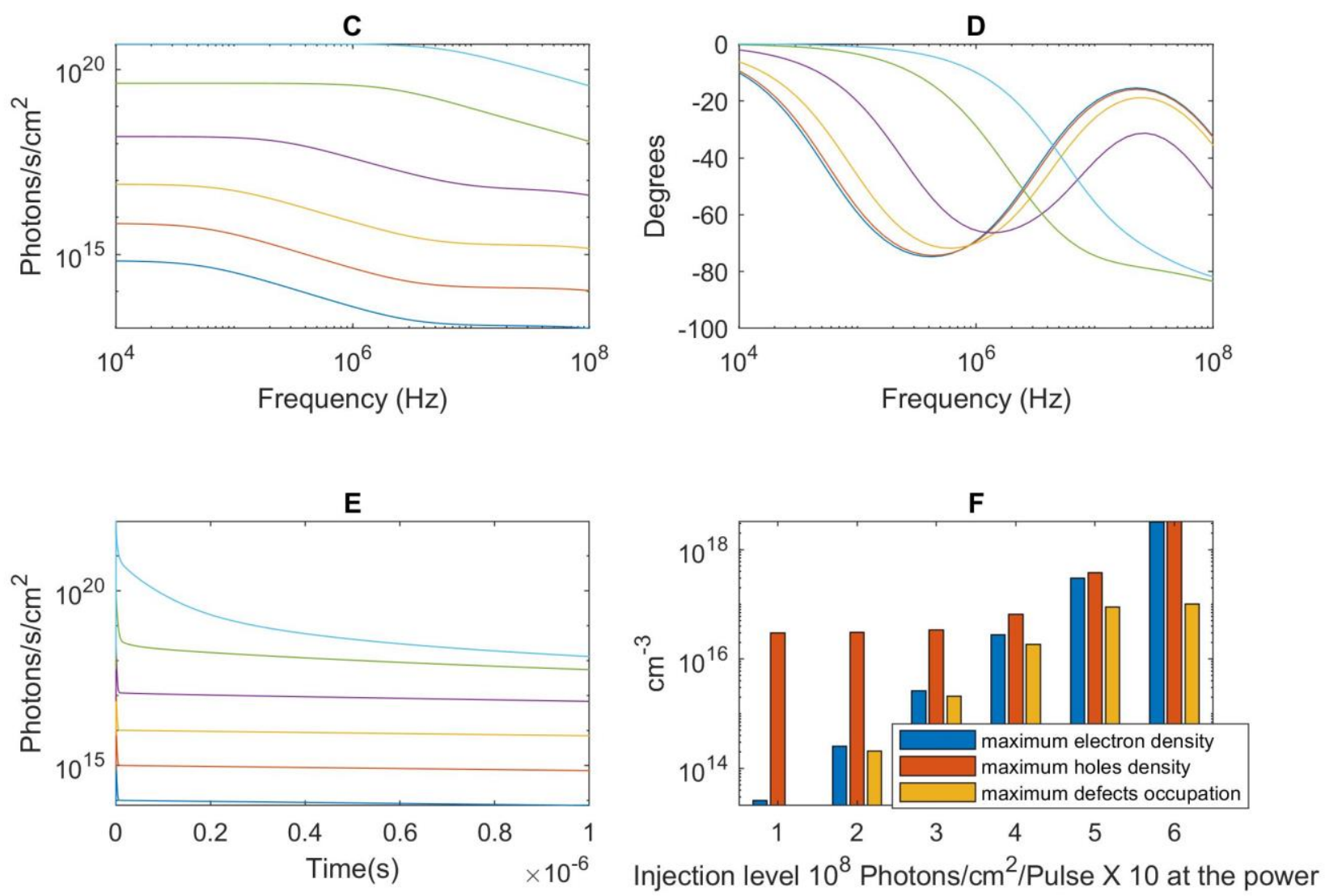

Fig. 3. Radiative recombination and shallow trapping. From A to D: simulated HF-MPL: A continuous part, B Injection level for electrons (blue), holes (red), trapped electrons (yellow), C first harmonic amplitude; D first harmonic phase at several values of the average excitation flux: $10^{15}$ photons $/ \mathrm{cm}^{2} / \mathrm{s}$ multiplied by ten at the power 1 (dark blue curve), 2 (red curve), 3 (yellow curve), 4 (purple curve), 5 (green curve), 6 (light blue curve). E and F: E simulated TRPL and F corresponding injection level corresponding to the pulsed excitation flux $10^{8} \mathrm{photons} / \mathrm{cm}^{2} / \mathrm{pulse}$ multiplied by ten at the power 1 (dark blue curve), 2 (red curve), 3 (yellow curve), 4 (purple curve), 5 (green curve), 6 (light blue curve). 

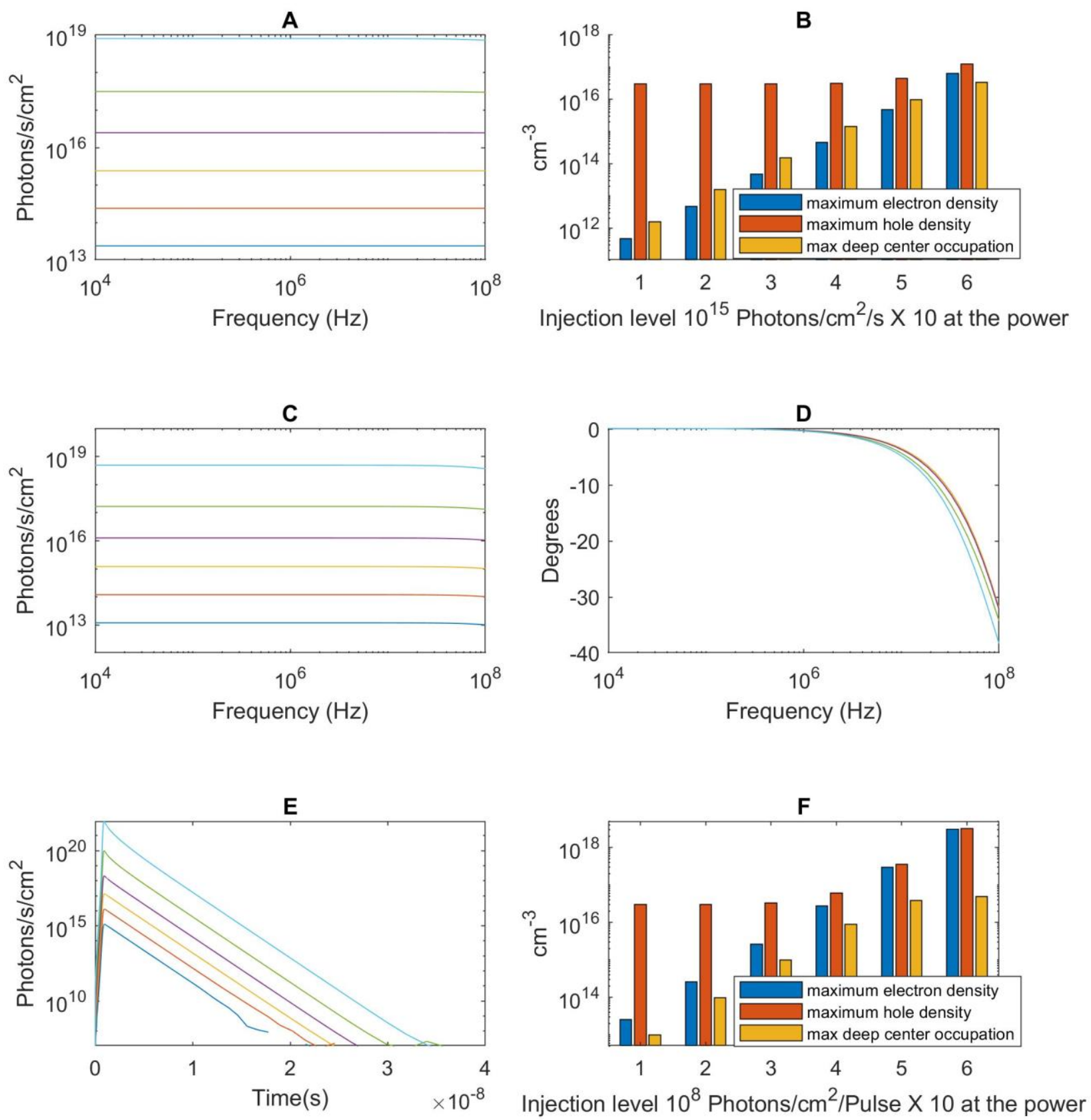

Fig. 4. Radiative recombination and deep mid-gap recombinant center. From A to D: simulated HF-MPL: A continuous part, B Injection level for electrons (blue), holes (red), trapped electrons (yellow), C first harmonic amplitude; D first harmonic phase at several values of the average excitation flux: $10^{15}$ photons $/ \mathrm{cm}^{2} / \mathrm{s}$ multiplied by ten at the power 1 (dark blue curve), 2 (red curve), 3 (yellow curve), 4 (purple curve), 5 (green curve), 6 (light blue curve). E and F: E simulated TRPL and F corresponding injection level corresponding to the pulsed excitation flux $10^{8}$ photons $/ \mathrm{cm}^{2} /$ pulse multiplied by ten at the power 1 (dark blue curve), 2 (red curve), 3 (yellow curve), 4 (purple curve), 5 (green curve), 6 (light blue curve). 
A

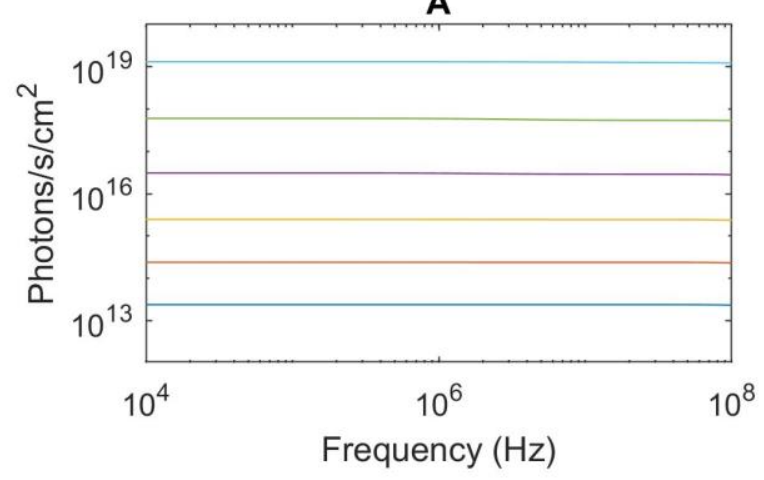

C

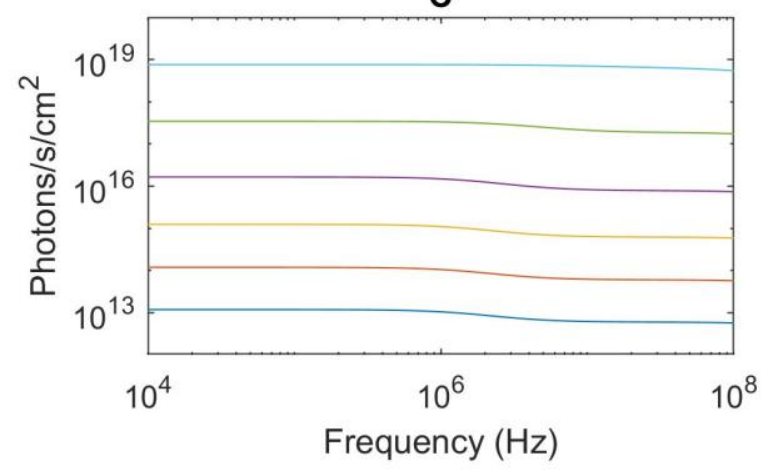

E

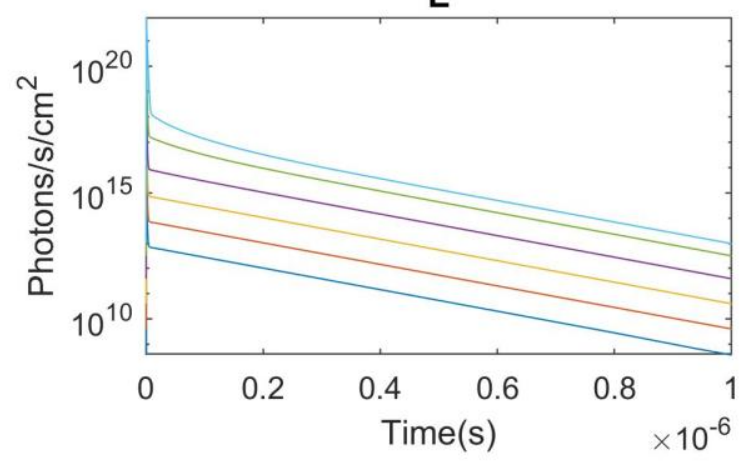

B

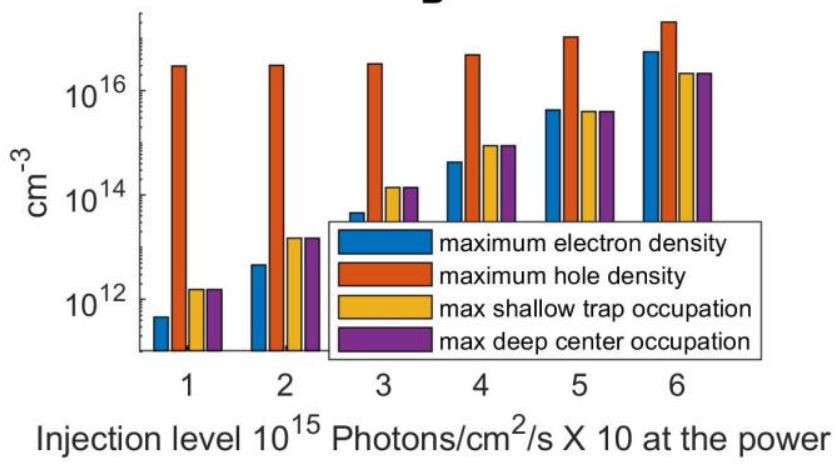

D

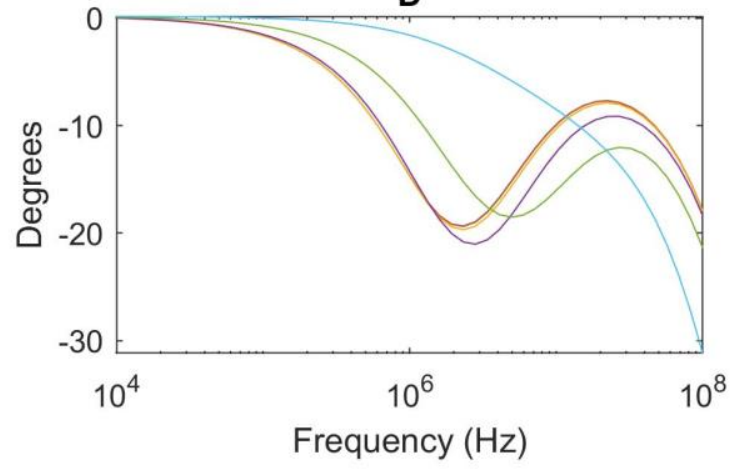

$\mathbf{F}$

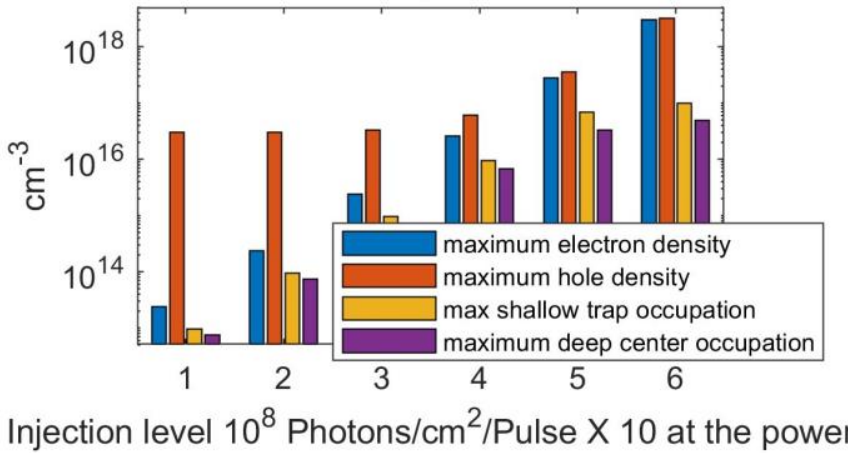

Fig. 5. All mechanisms together. From A to D: simulated HF-MPL: A continuous part, B Injection level for electrons (blue), holes (red), trapped electrons on defect 1 (yellow), trapped electrons on defect 2 (purple), C first harmonic amplitude; D first harmonic phase at several values of the average excitation flux: $10^{15}$ photons $/ \mathrm{cm}^{2} / \mathrm{s}$ multiplied by ten at the power 1 (dark blue curve), 2 (red curve), 3 (yellow curve), 4 (purple curve), 5 (green curve), 6 (light blue curve). E and F: E simulated TRPL and F corresponding injection level corresponding to the pulsed excitation flux $10^{8}$ photons $/ \mathrm{cm}^{2} /$ pulse multiplied by ten at the power 1 (dark blue curve), 2 (red curve), 3 (yellow curve), 4 (purple curve), 5 (green curve), 6 (light blue curve). 